\title{
Blood pressure targets in chronic kidney disease: Does proteinuria dictate how low we go?
}

\author{
Ainslie M. Hildebrand MD, Amit X. Garg MD PhD
}

See related research article by Lv and colleagues on page 949 and at www.cmaj.ca/lookup/doi/10.1503/cmaj.121468

$\mathrm{P}$ reventing kidney failure, cardiovascular events and death among patients with chronic kidney disease is important to patients, their care providers and health care systems, and blood pressure control is a fundamental part of the prevention strategy.

In December 2012, Kidney Disease: Improving Global Outcomes published a clinical practice guideline for the evaluation and management of chronic kidney disease, including statements on blood pressure control. ${ }^{1,2}$ These guidelines highlight lifestyle strategies to reduce blood pressure (e.g., decreasing salt intake, engaging in physical activity) and the preferential use of angiotensin-converting enzyme inhibitors or angiotensin-receptor blockers in some circumstances. Two other key statements in the guideline specifically pertain to the recommended target blood pressure in chronic kidney disease. These statements suggest that a target lower than 130/80 mm Hg may be appropriate for some patients with proteinuric chronic kidney disease, instead of the established target of lower than 140/90 $\mathrm{mm} \mathrm{Hg}$ that is recommended for the general population. However, the quality of evidence supporting a lower blood pressure target for patients with proteinuria was deemed to be low by the guideline committee.

In the research article related to this commentary, Lv and colleagues report the results of a systematic review of randomized controlled trials (RCTs) describing the effects of lower versus higher blood pressure targets in patients with chronic kidney disease. ${ }^{3} \mathrm{Lv}$ and colleagues ${ }^{3}$ summarized 11 RCTs that involved a total of 9287 patients with chronic kidney disease, defined as glomerular filtration rate $<60 \mathrm{~mL} / \mathrm{min}$ per 1.73 $\mathrm{m}^{2}$ or evidence of kidney damage. ${ }^{4}$ The targeted blood pressure levels in the groups receiving intensive blood pressure lowering varied across the trials, ranging from lower than $120 / 80 \mathrm{~mm}$ $\mathrm{Hg}$ to lower than $150 / 85 \mathrm{~mm} \mathrm{Hg}$, or to a mean arterial pressure of less than $92 \mathrm{~mm} \mathrm{Hg}$. The average difference in systolic pressure between the treatment and control arms of the trials was
$7.7 \mathrm{~mm} \mathrm{Hg}$; the average difference in diastolic pressure between the two arms was $4.9 \mathrm{~mm} \mathrm{Hg}$.

$\mathrm{Lv}$ and colleagues considered 3 types of outcome: cardiovascular events, all-cause mortality and kidney failure. They found no effect of intensive blood pressure lowering on cardiovascular outcomes or death, which they note may be due to the overall low number of events and lack of statistical power. Seven trials reported kidney failure events, and among the 5308 patients with chronic kidney disease included in these trials, the more intensive blood pressure-lowering strategy reduced the relative risk of kidney failure by $17 \%$ (hazard ratio [HR] $0.82,95 \%$ confidence interval $[\mathrm{CI}] 0.68-0.98, p=0.02$ ). In a subgroup analysis, this effect was modified by proteinuria status (urinary protein excretion $>300 \mathrm{mg} / \mathrm{d}$ or a protein-creatinine ratio $>$ $22 \mathrm{mg} / \mathrm{mmol}$ in a random urine sample). Among patients with proteinuria, the authors found a $27 \%$ reduction (HR $0.73,95 \%$ CI $0.62-0.86$ ) in kidney failure events with intensive blood pressure lowering, whereas no effect was seen in patients without proteinuria (HR 1.12, 95\% CI $0.67-1.87$ ). The prevention of kidney failure events seen in patients with proteinuria is the primary evidence upon which the target of $130 / 80 \mathrm{~mm} \mathrm{Hg}$ is based. ${ }^{1}$

Although Lv and colleagues focus on relative measures of benefit, absolute risk and absolute risk reduction warrant consideration. The absolute risk of kidney failure is influenced by many fac-

\section{- KEY POINTS}

- It is important to target a blood pressure of $140 / 90 \mathrm{~mm} \mathrm{Hg}$ or less in all patients with chronic kidney disease.

- Recent guidelines suggest potential value in a target blood pressure lower than $130 / 80 \mathrm{~mm} \mathrm{Hg}$ in patients with proteinuric chronic kidney disease for the purposes of preventing kidney failure (urine albumin-creatinine ratio $>3 \mathrm{mg} / \mathrm{mmol}$ in a spot urine sample); however, the exact target remains unclear.

- Because some patients with chronic kidney disease have a low absolute 10 -year risk of kidney failure, and using medications to target lower blood pressures carries a risk of adverse events, further evidence to guide clinical practice is needed. 
tors, including remaining life expectancy, the presence of diabetes, the glomerular filtration rate and the amount of proteinuria. ${ }^{5-7}$ Accordingly, for any given patient with chronic kidney disease, the absolute incidence of kidney failure may range from less than 1 per 1000 patient-years to almost 100 per 1000 patient-years. Assuming a relative risk reduction of $17 \%$ with intensive versus standard blood pressure control, for every 100 patients receiving treatment for 10 years, between 1 and 17 kidney failure events may be prevented. Considering the high cost of care for end-stage kidney disease and the associated poor outcomes for patients, the number of events that may be prevented in patients at high risk for disease progression is certainly important. However, the potential benefit of intensive blood pressure control is modest for patients who have a lower absolute risk.

Potential adverse events with intensive blood pressure control also warrant emphasis. Although Lv and colleagues did not find a significant increase in severe adverse events (including hypotension) or discontinuation of treatment associated with intensive blood pressure lowering, there was a high degree of heterogeneity with respect to which adverse events were reported among the 11 trials they included, and the data on hypotension were not suitable for meta-analysis. ${ }^{3}$ By nature of inclusion in a clinical trial, participants are highly selected for compliance to treatment and undergo strict monitoring. These patients typically have few comorbidities and are at lower risk for adverse events compared with the general population. Thus, widespread implementation of intensive blood pressure control in routine care for chronic kidney disease may have the unanticipated consequence of more drug-related adverse effects, hypotension and use of health services, especially among older patients and those with frailty, cardiovascular disease and autonomic neuropathy.

The systematic review by Lv and colleagues highlights important limitations in the underlying evidence. The data for lower blood pressure targets in proteinuric chronic kidney disease largely come from subgroup and post-hoc analyses of the Modification of Diet in Renal Disease study and the African American Study of Kidney Disease trial. ${ }^{8,9}$ Future studies should clarify the upper and lower targets for intensive blood pressure control in chronic kidney disease and the level of proteinuria at which they should be implemented. The Systolic Blood Pressure Intervention Trial is a large, multicentre, RCT sponsored by the US National Institutes of Health to compare the cardiovascular effects of systolic blood pressure targets below 120 and $140 \mathrm{~mm}$ $\mathrm{Hg}$ in adults with hypertension who do not have diabetes (ClinicalTrials.gov: NCT01206062). The results of this trial, expected by 2019 , could provide additional insight; the investigators aim to recruit a large proportion of patients with chronic kidney disease and perform a prespecified subgroup analysis by proteinuria. However, with the exclusion of patients with proteinuria greater than $1 \mathrm{~g} / \mathrm{d}$, this trial may not provide the highest-quality evidence to support current guidelines for intensive blood pressure control in some patients with chronic kidney disease.

\section{References}

1. (KDIGO) Blood Pressure Work Group. Kidney disease: improving global outcomes. KDIGO clinical practice guideline for the management of blood pressure in chronic kidney disease. Kidney Int Suppl 2012;2:337-414.

2. (KDIGO) CKD Work Group. Kidney disease; improving global outcomes. KDIGO 2012 clinical practice guideline for the evaluation and management of chronic kidney disease. Kidney Int Suppl 2013;3:1-150.

3. Lv J, Ehteshami P, Sarnak MJ, et al. Effects of intensive blood pressure lowering on the progression of chronic kidney disease: a systematic review and meta-analysis. CMAJ 2013;185:949-57.

4. National Kidney Foundation. K/DOQI clinical practice guidelines for chronic kidney disease: evaluation, classification and stratification. Am J Kidney Dis 2002;39(2 Suppl 1)S1-266.

5. Turin TC, Tonelli M, Manns BJ, et al. Lifetime risk of ESRD. J Am Soc Nephrol 2012;23:1569-78.

6. Fox CS, Matsushita K, Woodward M, et al. Associations of kidney disease measures with mortality and end-stage renal disease in individuals with and without diabetes: a meta-analysis. Lancet 2012;380:1662-73.

7. Gansevoort RT, Matsushita K, van der Velde M, et al. Lower estimated GFR and higher albuminuria are associated with adverse kidney outcomes. A collaborative meta-analysis of general and high-risk population cohorts. Kidney Int 2011;80: 93-104.

8. Peterson JC, Adler S, Burkart JM, et al. Blood pressure control, proteinuria, and the progression of renal disease. The Modification of Diet in Renal Disease study. Ann Intern Med 1995;123: 754-62.

9. Wright JT, Bakris G, Greene T, et al. Effect of blood pressure lowering and antihypertensive drug class on progression of hypertensive kidney disease: results from the AASK trial. JAMA 2002;288:2421-31.

Affiliations: Ainslie Hildebrande and Amit Garg are with the Division of Nephrology, and Amit Garg is with the Department of Clinical Epidemiology and Biostatistics, University of Western Ontario, London, Ont.

Contributors: Ainslie Hildebrand drafted the article, and both authors revised it critically for important intellectual content. Both authors contributed equally to the conception of ideas and independently approved the final version submitted for publication. 